\section{Bouter LM, Zielhuis GA, Zeegers MPA. Textbook of Epidemiology. Houten: Bohn Stafleu van Loghum; 2018.}

\author{
Carla Jorge Machado \\ (https://orcid.org/0000-0002-6871-0709) ${ }^{1}$ \\ Isabela Colem Castelo Borges \\ (https://orcid.org/0000-0002-0277-1875) ${ }^{2}$
}

${ }^{1}$ Faculdade de Medicina, Universidade Federal de Minas Gerais. Belo Horizonte MG Brasil.

${ }^{2}$ Universidade Federal de São João Del Rey. Divinópolis MG Brasil.

Há mais de 30 anos Raj Bhopal, professor emérito de Saúde Pública da Universidade de Edinburgh, Escócia, identificou o que chamou de uma 'epidemia de epidemiologia', cujo principal resultado visível era a crescente demanda de livros para uma audiência cada vez maior e mais diversa de estudantes ${ }^{1}$. Em revisão de 25 obras de epidemiologia introdutória, o autor propôs temas que não deveriam ser omitidos em uma obra dessa natureza, independente se for destinada a pesquisadores em geral, alunos de graduação ou de pós graduação ${ }^{1}$. Assim, em primeiro lugar, sugere que sejam abordados, em livros de epidemiologia, conceitos de (i) população, identificando a distinção entre causas individuais e coletivas das doenças; (ii) causalidade; (iii) história natural das doenças. Em segundo lugar, sugere ainda abordar os fundamentos e métodos relativos à (iv) padronização de taxas, sendo tal tópico um exemplo do controle dos fatores de confusão, e ao (v) estudo caso controle. Nesse último caso, Bhopal considera que o caso controle é uma referência e um ponto de partida para aferir quão adequadamente os métodos epidemiológicos são explicados de forma global em um livro ${ }^{1}$. Finalmente, dois tópicos importantes seriam aplicação da epidemiologia às informações de (vi) triagem ou rastreamento e (vii) assistência à saúde.

Examinados os principais aspectos de cada obra, Bhopal as incluiu em categorias: livros básicos para estudantes de graduação e profissionais com pouca experiência em epidemiologia; livros mais avançados para pós graduandos e profissionais com algum conhecimento na área; aqueles com ênfase em autoaprendizado, exercícios e aplicações práticas da epidemiologia; finalmente, livros que introduzem a epidemiologia à prática clínica.

Tendo por base esse arcabouço e na ausência de outros disponíveis, Textbook of Epidemiology é aqui avaliado sob este prisma. Utilizada por mais de três décadas em países de língua holandesa e, a partir de 2016, tendo passado por rigoroso processo de tradução para a língua inglesa, com impressão pela editora
Springer, a obra chamou nossa atenção. Direcionada a um público de acadêmicos, como estudantes de graduação, mestrado e doutorado, o livro conta com dez capítulos e adiciona bastante ao estudo e à compreensão da epidemiologia, pelos motivos expostos a seguir.

No que se refere ao conceito de população, a obra cumpre seu papel por deixar claro, no primeiro capítulo, que trata sobre o que é a epidemiologia e o desenvolvimento da disciplina deste os tempo de Hipócrates, no item 1.1, What is epidemiology, que a epidemiologia se dedica às populações humanas - ainda que exista um ramo, mencionado no livro, que deriva da epidemiologia na área de medicina veterinária. Ademais, no capítulo 2 , que aborda principalmente a definição de frequência de doença, suas medidas, além do conceito de tempo em epidemiologia, o item 2.3 - Types of population: cohort or dynamic population - distingue oportunamente entre coorte de acompanhamento e população dinâmica, distinção esta incomum em livros de epidemiologia. Assim, os autores explicam haver duas formas de se obter uma população: por meio de uma coorte, que muda com o tempo; ou definindo uma população dinâmica em um espaço, que não necessariamente muda com o passar do tempo. Auxilia a compreensão uma figura que apresenta gráficos que contrastam o tempo de duração da coorte e o tempo calendário comparativamente à evolução do tamanho populacional.

A causalidade é suficiente e oportunamente abordada. Há menção dos postulados de Koch e dos critérios de causalidade de Hill logo no capítulo 1, item 1.2 (Developments of epidemiology), deixando evidente que seguir preceitos epidemiológicos é buscar a causa. O que mais merece destaque, todavia, é, no capítulo 6, Etiology and causality, capítulo este que engloba essencialmente modelos de causalidade das doenças, a sinceridade com que os autores tratam a dificuldade em se estabelecer o que é a causa: no item 6.2.6, Causality at the individual versus the population level, após aplicarem o conceito de causa necessária à relação entre tabagismo e câncer de pulmão, concluem que dados populacionais podem ser traduzidos em riscos de doenças, mas não em conclusões definitivas sobre causalidade para um indivíduo específico. Enfim, os autores assumem a existência de uma dificuldade para os estudantes: o pensamento populacional não é simples e que a epidemiologia é uma ciência rigorosa².

Para explicar a história natural das doenças, figura que não contribui para a compreensão do tema é várias possibilidades para o curso da doença (tradução nossa). Infelizmente, a figura é muito complexa e deixa dúvidas aos leitores, sem devida explicação e

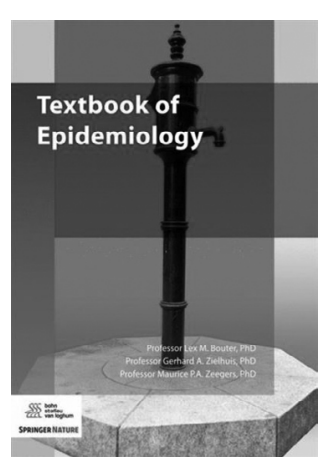


aprofundamento. Seria necessário que o conceito de história natural estivesse suficientemente claro para que se pudesse apreender o conteúdo do item 6.3.2, Prevention, que aborda as prevenções primária, secundária, e terciária. Dado que níveis de prevenção dependem da fase da história natural das doenças ${ }^{3}$, a ausência da explicação adequada desse tópico sem a devida relação com a prevenção é uma falha. Ainda, com o crescente risco de medicalização e condutas invasivas ${ }^{4}$, aliado ao envelhecimento da população e, em se tratando de obra que passou por recente revisão, é sentida a falta do conceito de prevenção quaternária ${ }^{4}$.

A padronização de taxas não possui tópico próprio, mas aparece associada às taxas brutas de mortalidade no item 2.5.3, Mortality rates: $a$ special type of incidence. A explicação e o exemplo apresentado são claros, inclusive traçando um paralelo entre método direto e indireto de forma simples - mas não simplista ou superficial.

Em se tratando do caso-controle, bem como de suas medidas específicas, especialmente o Odds Ratio, esta temática é apresentada de forma original e crítica no capítulo 3, o qual trata das relações entre a frequência de doenças e seus determinantes, além das medidas de associação e impacto de desfechos de saúde. Em se tratando mais detidamente do caso controle, a discussão é integrada ao fato de que, em um estudo de caso-controle não é possível calcular incidência - item 3.2.4., o odds ratio é uma medida conveniente (embora indireta e difícil de interpretar) quando não se dispõe da incidência (tradução nossa). Os autores retomam a ideia de população fechada (coorte) e dinâmica e, a partir dela, selecionam indivíduos doentes e saudáveis para compará-los. Auxilia muito o entendimento dos conceitos ilustração que mostra medidas de incidência relacionadas a variações na população dinâmica, ao tamanho populacional e ao tempo calendário. Assim, intuitivamente introduzem o que é um caso-controle aninhado versus um casocontrole mais comum, que vem de uma população dinâmica. Certamente, é um capítulo excelente. A seguir, no Capítulo 4, Study Design, são explicados os desenhos de estudo experimentais e observacionais e, nesse capítulo, é surpreendente a charge apresentada abordando a questão ética nos ensaios clínicos randomizados. A charge abre espaço para um pensamento crítico sobre os contextos de razoabilidade do uso do placebo.

A triagem ou rastreamento é explicada no contexto de teste diagnóstico, no Capítulo 9, Diagnostic and Prognostic Research, sem deixar margem para dúvidas. Ao nosso conhecimento, é o capítulo mais completo sobre teste diagnóstico. As figuras são elucidativas e nos resultados numéricos estão indicados os cálculos. Chama a atenção, de forma positiva, a explicação do kappa de Cohen (certamente um dos tópicos mais difíceis para entendimento dos estudantes), com apresentação gráfica inovadora.

Quanto à assistência à saúde, a temática é parcialmente abordada no Capítulo 10, Intervention, onde são relacionadas pesquisas que tratam de resultados de intervenções médicas. Nesse ponto fica a pergunta: por qual motivo os autores não elaboraram com maior detalhe a história natural da doença? Com vários exemplos de estudos conduzidos no mundo real, enfim, os autores enfatizam nesse capítulo todos os passos da pesquisa clínica experimental, e abordam também a questão dos efeitos colaterais de medicamentos, trazendo à tona, novamente, a estranha ausência da prevenção quaternária, que estaria alinhada com este capítulo.

Muito embora os próprios autores a considerem destinadas a acadêmicos, na classificação de Bhopal a obra Textbook of Epidemiology poderia ser classificada em qualquer das categorizações propostas, tendo em vista que a variedade de exercícios resolvidos a torna um livro acessível a qualquer interessado no tema.

Outro aspecto importante é que a epidemiologia é interdisciplinar e a correta análise de dados epidemiológicos exige que a comunicação e o trabalho em equipe seja essencial ${ }^{5}$. O capítulo 8 , Outbreak Epidemiology, introduz a lógica e a importância do trabalho em equipe, especialmente na investigação de surtos e epidemias. Espera-se que epidemiologistas trabalhem de forma conjunta na identificação de problemas de saúde pública com vistas à melhoria da saúde global ${ }^{5} \mathrm{e}$, desse modo, a obra também traz contribuição nesse sentido.

Os capítulos 5, Validity \& Reliability, e 7, Genetic Epidemiology, também trazem contribuições para além dos pontos básicos apontados por Bhopal e que são essenciais em um livro de epidemiologia. No capítulo 5, validade e confiabilidade são temáticas explicadas, talvez da forma mais abrangente dentre os livros conhecidos pelas autoras desta resenha. Além disso, o capítulo trata dos vários tipos de vieses que podem ocorrer em estudos epidemiológicos, da validade externa, e da calibração de instrumentos de medida. Já o capítulo 7 aborda, em linhas gerais, a interação entre fatores genéticos e ambientais e a implicação desta interação na saúde dos indivíduos. Explica em detalhes a importância dos estudos em famílias e como se deve proceder nesse tipo de investigação.

Finalmente, a preocupação em identificar livros textos adequados, suficientemente abran- 
gentes, e com explicações e exemplos que aproximem os alunos da disciplina epidemiologia e forneçam aos docentes opções para suas aulas é sempre justificada. A obra Textbook of Epidemio$\log y$, guardadas as devidas limitações e restrições aqui expostas, constitui-se em material inovador e de fácil compreensão. Uma versão na língua portuguesa seria bem-vinda.

\section{Referências}

1. Bhopal R. Which book? A comparative review of 25 introdutory epidemiology textbooks. J Epidemiol Community Health 1997; 51(6):612-622.

2. Keyes KM, Galea S. Current practices in teaching introductory epidemiology: how we got here, where to go. Am J Epidemiol 2014; 180(7):661-668.

3. Goswami S. Natural history of diseases and levels of prevention. 2017. 51 slides. [acessado 2018 Dez 31]. Disponível em: https://pt.slideshare.net/souravgoswamil1/ natural-history-of-disease-levels-of-prevention

4. Pandve HT. Changing concept of disease prevention: From primordial to quaternary. Arch Med Health Sci 2014; 2(2):254-256.

5. Goldmann E, Stark JH, Kapadia F, McQueen MB. Teaching Epidemiology at the Undergraduate Level: Considerations and Approaches. Am J Epidemiol 2018; 187(6):1143-1148. 
